Radovich, V.S. El mar también existe:reflexiones sobre la planificación espacial marina (PEM) como herramienta de gestión integral. Derecho y Ciencias Sociales. Mayo - Octubre 2020.N 23. Pgs 46-69 ISNN 1852-2971 Instituto de Cultura Jurídica y Maestría en Sociología Jurídica. FCJ y S. UNLP

\title{
El mar también existe: reflexiones sobre la planificación espacial marina (PEM) como herramienta de gestión integral
}

\author{
The Sea also exists. Reflections over marine spatial planning as an integrated management \\ tool
}

Violeta S. Radovich ${ }^{\bullet}$

\section{Resumen:}

El objetivo del presente artículo consiste en generar conocimiento en idioma español sobre la herramienta para la gestión integral del mar denominada "planificación espacial marina" (PEM). La metodología ha consistido en el análisis documental de guías de la Organización de las Naciones Unidas para la Educación, la Ciencia y la Cultura (UNESCO) que indican un método basado en pasos para instaurar la PEM en los países, de estudios académicos y de actas de foros de la temática en cuestión. Asimismo, se ha realizado un análisis de estudios de casos y se han identificado buenas prácticas y desafíos. Las conclusiones indican que es clave comprender profundamente el sistema de gobernanza actual e involucrar a los grupos de interesados en un diálogo constructivo.

Palabras clave: planificación, espacial, marina, ley, ambiental.

\begin{abstract}
:
The objective of this article is to generate knowledge in Spanish as regards the tool for integrated sea management known as "marine spatial planning" (MSP). Methodology consisted in the documentary analysis of guides elaborated by United Nations Educational, Scientific and Cultural Organization (UNESCO) that indicate a method based in steps to implement MSP in countries. Moreover, methodology has been based in the analysis of academic papers and deeds of forums regarding the issue. Conclusions show that it is essential to deeply understand the current governance system and to involve interested parties in a constructive dialogue.
\end{abstract}

Key words: marine, spatial, planning, environmental, law.

\footnotetext{
- Dra. en Derecho Marítimo, Especialista en Derecho Ambiental (UBA, FDER). Becaria posdoctoral CONICET- Investigadora Formada Instituto de Investigaciones Jurídicas y Sociales Ambrosio L. Gioja (UBA, FDER). Profesora Titular Escuela de Guerra Naval, Universidad Nacional de la Defensa (UNDEF). JTP de Derecho Ambiental (UBA, FDER), violetaradovich@derecho.uba.ar.
} 
Radovich, V.S. El mar también existe:reflexiones sobre la planificación espacial marina (PEM) como herramienta de gestión integral. Derecho y Ciencias Sociales. Mayo - Octubre 2020.N ${ }^{\circ} 23$. Pgs 46-69 ISNN 1852-2971 Instituto de Cultura Jurídica y Maestría en Sociología Jurídica. FCJ y S. UNLP

\section{El mar también existe: reflexiones sobre la planificación espacial marina (PEM) como herramienta de gestión integral}

Violeta S. Radovich

"La función del arte / 1

Diego no conocía la mar. El padre, Santiago Kovadloff, lo llevó a descubrirla.

Viajaron al sur.

Ella, la mar, estaba más allá de los altos médanos, esperando.

Cuando el niño y su padre alcanzaron por fin aquellas cumbres de arena, después de mucho caminar, la mar estalló ante sus ojos. Y fue tanta la inmensidad de la mar, y tanto su fulgor, que el niño quedó mudo de hermosura.

Y cuando por fin consiguió hablar, temblando, tartamudeando, pidió a su padre: - ¡Ayúdame a mirar!"

Eduardo Galeano, El libro de los abrazos.

\section{Introducción}

Cicin-Sain, Vanderzwaag \& Balgos (2015) citados por OECD (2016, p. 225) sostienen que la gestión integral implica procesos para coordinar las acciones de las diversas agencias gubernamentales involucradas en los asuntos oceánicos. La denominada gestión integral del mar propone una visión omnicomprensiva, por oposición a su gestión sectorizada, es decir aquella que se enfoca de manera autónoma en determinado sector sin interrelación entre ellos, por ejemplo el de la pesca o el del turismo o el de los hidrocarburos, o más cercano en el tiempo, en la generación de energías renovables en el mar o en la minería marina o en la prospección de recursos genéticos marinos (Radovich, 2018). En el mismo sentido, el último informe presentado por IPBES (Plataforma Intergubernamental Científico-normativa sobre Diversidad Biológica y Servicios de los Ecosistemas) en 2019 plantea que se debe alcanzar la gobernanza "integrativa" para atacar la incoherencia sectorial en las políticas. La gobernanza integrativa se plantea como uno de los componentes para alcanzar la gobernanza "transformadora", junto con la gobernanza informada, adaptativa e inclusiva (IPBES, 2019).

La planificación espacial marina (PEM) ha sido definida como un proceso público de análisis y de distribución espacial y temporal de las actividades humanas en áreas marinas para alcanzar objetivos ecológicos, económicos y sociales, que suelen especificarse a través de un proceso político (Ehler \& Douvere, 2009). 
Radovich, V.S. El mar también existe:reflexiones sobre la planificación espacial marina (PEM) como herramienta de gestión integral. Derecho y Ciencias Sociales. Mayo - Octubre 2020.N 23. Pgs 46-69 ISNN 1852-2971 Instituto de Cultura Jurídica y Maestría en Sociología Jurídica. FCJ y S. UNLP

La PEM se implementa porque existen o se prevé que existan usos incompatibles o usos que afecten adversamente importantes áreas naturales. Los problemas suelen estar relacionados con la compatibilización entre el desarrollo económico (por ejemplo, dónde permitir la instalación de estructuras de energía renovable o estructuras de acuacultura) y la conservación del ambiente (por ejemplo, qué áreas biológicas y ecológicas importantes necesitan ser protegidas). En este sentido, Bélgica y la República Federal de Alemania iniciaron la PEM luego de preguntarse sobre la ubicación de las nuevas estructuras de energía eólica en el mar.

La manera en que se gestiona el Océano en un lugar del mundo impacta considerablemente en otras regiones, por lo que se necesita un enfoque holístico global como la PEM. La PEM también consiste en afrontar los desafíos del futuro, por ejemplo la alimentación de la población mundial, el desarrollo de la seguridad energética o la lucha contra el cambio climático. En mayo de 2018 en el Primer Foro Internacional de Planificación Espacial Marina celebrado en Bruselas se recomendó que se desarrollen guías de PEM transfronteriza basadas en un consenso global de estándares y buenas prácticas. Se sostuvo que la PEM combina dimensiones humanas, económicas y ecológicas; además de constituir un gran ejercicio y una gran oportunidad para que la ciencia informe a la política (EC DG MARE, UNESCO IOC, 2018).

Jones, Lieberknecht \& Qiu (2016) explican que la planificación espacial en la tierra ha sido evaluada al menos desde 1970, con los trabajos de Alterman \& Hill (1978) y Calkins (1979). Las iniciativas sobre la PEM se han multiplicado de 5 países en 2005 a alrededor de 90 en 2017. Estas iniciativas están en línea con los esfuerzos por alcanzar el Objetivo de Desarrollo Sostenible (ODS) 14 de la Agenda de Desarrollo Sostenible 2030 (Organización de las Naciones Unidas, 2015) denominado "Vida debajo del agua", cuyo fin es la conservación y el uso sostenible de los océanos. El objetivo común consiste en cubrir más de la tercera parte de la Zona Económica Exclusiva (ZEE) mundial con planes espaciales marinos aprobados para el año 2030 (Iglesias Campos, 2018). En este sentido, el Proyecto Seabed 2030 aspira a recolectar la totalidad de los datos batimétricos disponibles con el objetivo de producir el mapa definitivo del fondo marino mundial para 2030 y ponerlo a disposición de todo el público ${ }^{1}$.

\footnotetext{
${ }^{1}$ Proyecto Seabed 2030, Recuperado de https://seabed2030.gebco.net/
} 
Radovich, V.S. El mar también existe:reflexiones sobre la planificación espacial marina (PEM) como herramienta de gestión integral. Derecho y Ciencias Sociales. Mayo - Octubre 2020.N 23. Pgs 46-69 ISNN 1852-2971 Instituto de Cultura Jurídica y Maestría en Sociología Jurídica. FCJ y S. UNLP

En Europa, la legislación sobre la PEM (Directiva PEM, 2014) ${ }^{2}$ establece que se debe propender a la colaboración transfronteriza entre los Estados Miembro para establecer planes espaciales marinos coherentes, y no sólo entre los Estados Miembro; sino también con países que no pertenezcan a la Unión Europea (UE). Desde 2014 en la UE, se financiaron 12 proyectos transfronterizos para desarrollar herramientas e instrumentos con el objetivo de apoyar la implementación de la PEM en todas las cuencas marinas (Mabilia, 2018). Por ejemplo, el Proyecto de la UE denominado MESMA (Monitoring and Evaluation of Spatially Managed Areas) que se extendió desde los años 2009 a 2013 se ha enfocado en la PEM y su objetivo ha sido producir herramientas de manejo integrado (conceptos, modelos y guías) para monitorear, evaluar e implementar las áreas de manejo marino espacial $^{3}$.

\section{La planificación espacial marina}

Ehler \& Douvere (2009) describen los pasos que deberían seguirse para establecer un proceso de PEM. El primer paso consiste en designar a la Autoridad que se encargará de llevar adelante la PEM. El segundo paso implica la obtención del apoyo financiero. El tercero, la organización del proceso a través de la pre-planificación y el cuarto, en organizar la participación de los grupos de interesados; es decir, quiénes, dónde y cómo participarán. A continuación, el quinto paso se trata de definir y analizar las condiciones ecológicas, ambientales y oceanográficas, las actividades humanas y los conflictos existentes actuales. El sexto paso consiste en definir y analizar las condiciones futuras, el séptimo en preparar y aprobar el plan de manejo espacial, el octavo en implementarlo y ejecutarlo y el noveno, en monitorearlo y evaluar su performance. Por último, el décimo paso implica adaptar el proceso de manejo marino espacial.

También se han planteado críticas a este método paso a paso, se ha dicho que a pesar de que se define a la PEM como un proceso enfocado en lograr una diversidad de objetivos ecológicos, económicos y sociales, la realidad es que muchos procesos de PEM suelen iniciarse y guiarse por objetivos sectoriales específicos, en general relacionados con procesos energéticos. Por lo que se ha sostenido que en lugar de "planificación espacial

\footnotetext{
${ }^{2}$ Directiva 2014/89/EU del Parlamento Europeo y del Consejo del 23 de julio de 2014, por la que se establece un marco para la ordenación del espacio marítimo, Diario Oficial de la Unión Europea L 257/135. Recuperado de https://eur-lex.europa.eu/legal-content/ES/TXT/PDF/?uri=CELEX:32014L0089\&from=EN

3 Proyecto MESMA (Monitoring and Evaluation of Spatially Managed Areas), Recuperado de http://mesma.org/default.asp?ZNT=S0T1O474
} 
Radovich, V.S. El mar también existe:reflexiones sobre la planificación espacial marina (PEM) como herramienta de gestión integral. Derecho y Ciencias Sociales. Mayo - Octubre 2020.N 23. Pgs 46-69 ISNN 1852-2971 Instituto de Cultura Jurídica y Maestría en Sociología Jurídica. FCJ y S. UNLP

marina", el instrumento debería llamarse "planificación sectorial estratégica" (Jones et al., 2016).

Asimismo, se ha dicho que este proceso paso a paso no suele aplicarse en la práctica y que la PEM suele comprender un enfoque verticalista, que el proceso de toma de decisiones entre los grupos de interés y los decisores no es colaborativo. Se argumenta que se implementan plataformas de participación, pero luego se desconectan de las tomas de decisiones finales (Jones et al., 2016).

\section{A continuación, se desarrollan los pasos que propone la Guía UNESCO:}

Paso 1: Designar a la Autoridad que desarrollará la PEM

Una manera de designar a la autoridad encargada de llevar adelante la PEM es mediante la sanción de una nueva ley; tal como realizó el Reino Unido a través de la ley denominada The Marine and Coastal Access Act ${ }^{4}$. A través de esta ley se estableció una nueva autoridad llamada Marine Management Organization para desarrollar planes espaciales marinos. Un enfoque similar se adoptó en Australia donde una nueva ley creó la Autoridad llamada Great Barrier Reef Marine Park Authority ${ }^{5}$. En 2008, el Estado de Massachusets en EE.UU. sancionó la ley denominada New Oceans Act ${ }^{6}$ que también designo a la autoridad encargada de desarrollar la PEM.

En la mayoría de las iniciativas a nivel mundial sobre la PEM, se suele designar una nueva autoridad para desarrollarla, mientras que la implementación se lleva adelante mediante autoridades e instituciones ya existentes.

Otra manera de designar a la Autoridad que llevará adelante la PEM consiste en partir de legislación ya existente, ya sea re-interpretándola o modificándola ligeramente para proveer una base para desarrollar la PEM. Esta legislación puede ser la referida a la gestión costera integrada, a la explotación y la exploración del Mar Territorial o la Zona Económica Exclusiva, o a la protección del ambiente marino.

\footnotetext{
4 The Marine and Coastal Acess Act, del 12 de noviembre de 2009, c. 23. Recuperado de http://www.legislation.gov.uk/ukpga/2009/23/pdfs/ukpga_20090023_en.pdf

5 The Great Barrier Reef Marine Park Act, 1975, Federal Register of Legislation C2017C00279. Recuperado de https://www.legislation.gov.au/Details/C2017C00279

${ }^{6}$ Oceans Act of 2008, Chapter 114, Commonwealth of Massachusetts, United States of America. Recuperado de https://malegislature.gov/Laws/SessionLaws/Acts/2008/Chapter114
} 
Radovich, V.S. El mar también existe:reflexiones sobre la planificación espacial marina (PEM) como herramienta de gestión integral. Derecho y Ciencias Sociales. Mayo - Octubre 2020.N 23. Pgs 46-69 ISNN 1852-2971 Instituto de Cultura Jurídica y Maestría en Sociología Jurídica. FCJ y S. UNLP

En los Países Bajos, la PEM se desarrolló a través de "un organismo inter-ministerial de consulta para el Mar del Norte", compuesto por representantes de todos los ministerios relevantes, como el de Defensa, Transporte, Obras Públicas y Gestión del Agua, Economía y el de Ambiente. En Noruega, se utilizó un enfoque similar y el organismo fue presidido por el Ministro de Ambiente.

Otra manera de designar la autoridad para desarrollar la PEM es agregar disposiciones a los proyectos de ley en análisis. En algunos países, la legislación para regular la nueva infraestructura en el mar, ya sea las estructuras de energía renovable y la acuacultura está aún en progreso. Se pueden incorporar, por ejemplo, disposiciones que establezcan que la PEM sea obligatoria, por ejemplo, cuando se otorgan las licencias para instalar estas estructuras.

\section{Paso 2: El Financiamiento}

Este paso consiste en lograr un plan financiero que estime los costos de las actividades de la PEM y en identificar medios alternativos para obtener financiamiento._Se argumenta que la clave del éxito es contar con múltiples fuentes de financiamiento y no confiar sólo en una (Ehler \& Douvere, 2009). Estos mecanismos pueden incluir, por ejemplo, subvenciones y donaciones de organizaciones internacionales y multinacionales, subvenciones de fundaciones, colaboraciones de organizaciones no-gubernamentales, fondos del sector privado y pagos por los servicios ambientales que presta el mar. Dependiendo del contexto, no todos los mecanismos de financiamiento alternativos son igual de viables. La elección del tipo de mecanismo de financiamiento debe basarse en una serie de consideraciones financieras, legales, administrativas, sociales, políticas y ambientales.

Se recomienda establecer la obligatoriedad de los mecanismos financieros en la legislación, para asegurar que el proceso de la PEM no peligre por falta de recursos.

\section{Paso 3: La pre-planificación}

En la pre-planificación se deberían establecer: (1) el equipo que llevará adelante la PEM; (2) el plan de trabajo (en el que se debe incluir el cronograma); (3) el plazo temporal para la planificación; (4) el conjunto de principios que serán aplicables; (5) las metas a lograr; (6) 
Radovich, V.S. El mar también existe:reflexiones sobre la planificación espacial marina (PEM) como herramienta de gestión integral. Derecho y Ciencias Sociales. Mayo - Octubre 2020.N 23. Pgs 46-69 ISNN 1852-2971 Instituto de Cultura Jurídica y Maestría en Sociología Jurídica. FCJ y S. UNLP

los objetivos de manera clara y mensurable y (7) la evaluación de los riesgos y las posibles contingencias durante el proceso de planificación.

Es esencial desarrollar un plan de trabajo que especifique qué etapas del proceso deberían ser desarrolladas por qué parte, en qué momento, a qué costo y cómo las partes interrelacionan entre sí.

La PEM suele ser más efectiva si se realiza con un "enfoque basado en el objetivo". A modo ilustrativo, el plan de manejo oceánico canadiense denominado Canada's Eastern Scotian Shelf integrated ocean management plan ${ }^{7}$ aplica este enfoque.

Con respecto al plan de trabajo, Ehler \& Douvere (2009) enumeran las acciones que se deberían seguir:

(1) Listar las actividades principales que se necesitan para desarrollar el plan;

(2) Separar cada actividad en tareas pequeñas realizables, por ej. una tarea que pueda ser gestionada por un individuo o grupo y que sea fácil de visualizar en término de los recursos que se necesiten y el tiempo que lleve completarlas. Sin embargo, hay que ser cuidadoso y no separar las actividades en componentes muy pequeños;

(3) Elegir períodos de tiempo apropiados para especificar cuándo se desarrollarán las actividades (semanal, mensual o quincenalmente);

(4) Clarificar la secuencia y las relaciones entre las tareas (¿Hay alguna tarea que deba ser completada antes de que se pueda comenzar con otra actividad? ¿Hay dos tareas que puedan ser realizadas al mismo tiempo?);

(5) Estimar el comienzo y la duración de cada actividad, que puede representarse a través de una línea o barra en un cuadro;

(6) Identificar los eventos principales (hitos) para ayudar a monitorear el progreso. Suelen ser fechas en las cuales las tareas deben ser completadas; $y$

(7) Asignar responsabilidades sobre las tareas entre los varios miembros del equipo de la PEM.

\footnotetext{
${ }^{7}$ Eastern Scotian Shelf Integrated Ocean Management Plan, Government of Canada, 2007, Recuperado de www.mar.dfo-mpo.gc.ca/oceans/e/essim/essim-intro-e.html
} 
Radovich, V.S. El mar también existe:reflexiones sobre la planificación espacial marina (PEM) como herramienta de gestión integral. Derecho y Ciencias Sociales. Mayo - Octubre 2020.N 23. Pgs 46-69 ISNN 1852-2971 Instituto de Cultura Jurídica y Maestría en Sociología Jurídica. FCJ y S. UNLP

La PEM debería ser guiada por un conjunto de principios, a continuación se describen algunos de los principios:

El principio de integridad ecosistémica: implica poner el foco principal en mantener la estructura del ecosistema y su funcionamiento dentro de un área de planificación marina espacial. Incluye reconocer que los ecosistemas son dinámicos, cambiantes y que a veces no se los comprende acabadamente (por lo que requieren que la toma de decisiones sea precautoria).

El principio de integración: trabajar en compartimentos sectoriales e institucionales crea costos por falta de coordinación que deben ser identificados y solucionados. La PEM puede jugar un rol crítico para facilitar la coherencia y la integración. La integración entre los distintos niveles de gobierno puede ayudar a tomar decisiones y acciones complementarias y que los distintos sectores se refuercen mutuamente.

El principio de fideicomiso público: implica que el espacio marino y los recursos marinos pertenecen al pueblo y permanecen en fideicomiso para el pueblo y las futuras generaciones. El espacio marino debería ser gestionado como "un bien común", como parte del dominio público, no apropiado exclusivamente por ningún grupo o interés privado.

El principio de transparencia: los procesos utilizados para tomar decisiones deben ser fácilmente comprendidos por el público, se le debe permitir a los ciudadanos observar el proceso de toma de decisiones, cómo se distribuyen los recursos, y cómo se llegó a las decisiones que afectan sus vidas.

El principio precautorio: si una decisión puede causar peligro de daño grave o irreversible al ambiente, y no existe certeza científica acerca de que el daño no ocurrirá, la carga de la prueba cae en aquellos que decidieron tomar la decisión.

El principio contaminador-pagador: los daños por contaminación al ambiente deben ser pagados por la parte responsable de su generación.

En este paso también se deben establecer las metas y los objetivos. Las siguientes son las diferencias entre las metas y los objetivos que señalan los autores. La diferencia entre estos reside en que las primeras son generales, no son mensurables; los segundos son específicos y son mensurables. Por ejemplo, una meta sería conservar y proteger los recursos marinos; un objetivo sería implementar una red representativa de áreas marinas protegidas (AMPs) antes de 2021. 
Radovich, V.S. El mar también existe:reflexiones sobre la planificación espacial marina (PEM) como herramienta de gestión integral. Derecho y Ciencias Sociales. Mayo - Octubre 2020.N 23. Pgs 46-69 ISNN 1852-2971 Instituto de Cultura Jurídica y Maestría en Sociología Jurídica. FCJ y S. UNLP

\section{Paso 4: Participación de los grupos de interesados}

La participación de los grupos de interesados en la iniciativa de la PEM debe estar guiada por dos preguntas: (a) ¿Quién decide durante la planificación y los pasos de implementación de la PEM? y (b) ¿Quién es el responsable de la planificación y el desarrollo de la PEM?

Por ejemplo, quizás ya haya en el sistema normativo la obligación legal que establezca que se deben compartir las decisiones sobre las inversiones en el mar a largo plazo con los grupos de interesados o ciertos grupos de interesados (por ej. las comunidades originarias) o quizás haya una obligación legal que establezca la realización de una consulta pública sobre el plan espacial antes de su implementación. En caso de no existir estas obligaciones legales, es importante definir cómo debería ser la participación de los grupos de interesados para lograr un resultado exitoso.

En la República Argentina, la participación es obligatoria según el artículo 20 de la Ley General del Ambiente (LGA) que plantea que las autoridades deberán realizar las audiencias o consultas públicas como instancias obligatorias antes de la autorización de aquellas actividades que pueden generar efectos negativos y significativos sobre el ambiente. La opinión u objeción de los participantes no será vinculante para las autoridades convocantes, pero en caso que las autoridades presenten opinión contraria a los resultados alcanzados en la audiencia pública, deberán fundamentarla y hacerla pública. Se puede citar el caso Villivar, en el que la Corte Suprema de Justicia Argentina anuló el funcionamiento de una mina porque no se había realizado la audiencia pública antes de su puesta en funcionamiento.

Involucrar a los grupos de interesados claves en el desarrollo de la PEM es esencial por un número de razones. El objetivo más importante por el que se debe involucrar a los grupos de interés se basa en que la PEM intenta lograr múltiples objetivos (sociales, económicos y ecológicos) y debería entonces reflejar la mayoría de las expectativas, oportunidades y conflictos que ocurren en el área de la PEM.

La participación de los grupos de interesados difiere según el país y suele ser influida culturalmente. Sin embargo, involucrar a demasiados grupos de interesados en el momento inadecuado o de una forma incorrecta puede consumir mucho tiempo y distraer del resultado esperado. Para involucrar a los grupos de interesados eficazmente (es decir con una orientación a los resultados esperados) y eficientemente (llegar a los resultados esperados al mínimo costo), se deberían considerar tres preguntas importantes (Ehler \& Douvere, 2009): 
Radovich, V.S. El mar también existe:reflexiones sobre la planificación espacial marina (PEM) como herramienta de gestión integral. Derecho y Ciencias Sociales. Mayo - Octubre 2020.N 23. Pgs 46-69 ISNN 1852-2971 Instituto de Cultura Jurídica y Maestría en Sociología Jurídica. FCJ y S. UNLP

Pregunta 1. ¿Quiénes deberían estar involucrados?

Pregunta 2. ¿Cuándo deberían estar involucrados?

Pregunta 3. ¿Cómo deberían estar involucrados?

Waldmann et al. (2019) sostienen que la consulta es un método formal, por oposición a la cooperación, que es un método informal y que es importante asentar por escrito lo que surja en la etapa de consulta para poder invocarlo, si fuera necesario, en una etapa posterior.

\section{Pregunta 1: Definir quiénes deberían estar involucrados en la PEM}

Entre los individuos, grupos $\mathrm{u}$ organizaciones que deberían ser considerados para involucrarse en la PEM se deberían incluir a aquellos que:

- serán afectados por las decisiones de la PEM;

- dependen de los recursos del área de manejo donde se tomarán las decisiones sobre la PEM;

- tienen o pueden tener demandas legales sobre el área de manejo;

- realizan actividades que impactan en áreas o recursos del área de manejo;

- tienen intereses especiales estacionales o geográficos en el área de manejo; y

- tienen un interés especial en el manejo del área (por ej. Ong’s ambientales y grupos de promoción cultural).

\section{Pregunta 2: Definir cuándo deberían estar involucrados en la PEM}

Idealmente, se deben involucrar desde el inicio del proceso y de manera continuada y sostenida durante todo el proceso.

a. Participación en la pre-planificación y planificación de la PEM

Durante las fases de la pre-planificación y la planificación de la PEM (Pasos 3, 5 y 6) es beneficioso incluir a la mayor cantidad de grupos de interesados posible, ya que de este modo se permitirá recabar información sobre un gran rango de expectativas, oportunidades y conflictos que tienen lugar en el área de manejo. El Estado de Massachusetts en EE.UU., por ejemplo, organizó 18 audiencias públicas, la información obtenida proveyó una base para identificar las metas y los objetivos de la iniciativa sobre la PEM. 
Radovich, V.S. El mar también existe:reflexiones sobre la planificación espacial marina (PEM) como herramienta de gestión integral. Derecho y Ciencias Sociales. Mayo - Octubre 2020.N 23. Pgs 46-69 ISNN 1852-2971 Instituto de Cultura Jurídica y Maestría en Sociología Jurídica. FCJ y S. UNLP

También se puede mencionar el desarrollo del plan maestro belga para el Mar del Norte, que tomó un nuevo enfoque (luego de que fallaran intentos iniciales), comenzó con un período de seis meses en el que se realizaron entrevistas y reuniones continuadas con diferentes sectores y grupos de interesados.

\section{b. Desarrollo del plan de la PEM}

Se debería seleccionar a un grupo de interesados principal para que participe en el análisis y en la selección de las alternativas del plan y en las consecuencias que traerán aparejadas las diferentes alternativas en las áreas de su respectivo interés (Paso 7). Tanto Bélgica, la República Federal de Alemania y los Países Bajos pusieron a disposición un plan borrador de PEM para la consulta pública. Este período suele abarcar entre tres y seis meses, y en algunos casos hasta un año.

c. Monitoreo y evaluación de la performance de la PEM

También se debería involucrar a los grupos de interesados en la evaluación del cumplimiento general en el logro de las metas y los objetivos de los planes y las medidas de la PEM.

Existen diversas maneras de involucrar a los grupos de interesados, desde la "comunicación" sin participación real, a la "negociación" donde el poder de toma de decisiones se comparte entre los grupos de interesados.

Por ejemplo, las autoridades alemanas publicaron los borradores de la PEM para el Mar del Norte y el Mar Báltico que estuvieron disponibles para la consulta pública por un período de cuatro meses. El Reino Unido presentó el borrador de su Proyecto de Ley Sobre Acceso Marino y Costero que estuvo disponible durante tres meses para la consulta pre-legislativa antes de presentarlo en el Congreso.

\section{Paso 5: Definición y análisis de las condiciones ecológicas y oceanográficas, las actividades humanas y los conflictos existentes actuales}

Los siguientes son los resultados que deberían obtenerse de este paso: un inventario y mapas de las áreas de importancia biológica y ecológica en el área de manejo marina; un inventario y mapas de las actividades humanas actuales (y las presiones) en el área de manejo marina; 
Radovich, V.S. El mar también existe:reflexiones sobre la planificación espacial marina (PEM) como herramienta de gestión integral. Derecho y Ciencias Sociales. Mayo - Octubre 2020.N 23. Pgs 46-69 ISNN 1852-2971 Instituto de Cultura Jurídica y Maestría en Sociología Jurídica. FCJ y S. UNLP

una evaluación de los posibles conflictos y las compatibilidades entre los usos humanos; y una evaluación de los posibles conflictos entre los usos humanos actuales y el ambiente.

Se deberían considerar las siguientes preguntas al preparar el inventario: ¿Cuáles son las características ecológicas específicas del área de manejo marina?, ¿Dónde están ubicadas las áreas particularmente sensibles o ecológicas?, ¿Hay factores económicos y sociales específicos que deberían ser considerados?, ¿Hay algún sector que dependa de algún tipo de área marina en particular?, ¿Cuáles son las principales presiones y amenazas en el área de manejo marino?, ¿Cuáles son las mayores fortalezas que pueden moldear el desarrollo marino en el futuro cercano? (Ehler \& Douvere, 2009)

Con respecto a la recolección y al mapeo de la información sobre las condiciones ecológicas, ambientales y oceanográficas, se recomienda guiarse por los criterios para identificar áreas marinas ecológica o biológicamente significativas establecidos por la Convención sobre la Diversidad Biológica (CDB).

Entre los ejemplos de estas áreas biológicas o ecológicas importantes se incluyen: áreas de alta biodiversidad, áreas de alto endemismo (especies, poblaciones o comunidades), áreas de alta productividad (especies, poblaciones o comunidades). Entre estas últimas se incluyen: áreas de surgencia, áreas de reproducción, áreas de parto, áreas de alimentación, áreas de anidación, áreas de cría, áreas de descanso, puntos de escalas migratorios, humedales, manglares, arrecifes de coral.

En lo concerniente a la recolección y mapeo de la información sobre las actividades humanas, se detallan a continuación algunos tipos de usos humanos de las áreas marinas: pesca comercial: con redes, anzuelos/líneas, trampas, arpones, redes de arrastre, redes de cerco, redes de arrastre de playa; aquicultura/maricultura; pesca recreativa: con anzuelos/líneas, pesca submarina; recreación: navegación a vela, en bote, artefactos para el agua personales, buceo/snorkeling, avistaje de fauna marina; transporte marino: buques de carga, tanqueros, transportadores de gas licuado natural (LNG), cruceros, ferries; operaciones portuarias: dragado, disposición del material de dragado, aeropuertos marinos, instalaciones de producción industrial en el mar, terminales de gas licuado natural (LNG), exploración en el mar de hidrocarburos y gas), cables, líneas de transmisión, minería de arena; energía renovable en el mar: parques eólicos, parques mareomotrices, plantas de desalinización oceánicas, sitios de secuestro de carbón, operaciones militares, reservas 
Radovich, V.S. El mar también existe:reflexiones sobre la planificación espacial marina (PEM) como herramienta de gestión integral. Derecho y Ciencias Sociales. Mayo - Octubre 2020.N 23. Pgs 46-69 ISNN 1852-2971 Instituto de Cultura Jurídica y Maestría en Sociología Jurídica. FCJ y S. UNLP

marinas estrictamente protegidas, parques marinos de múltiples usos, investigación científica, conservación cultural e histórica.

Un aspecto de la PEM que puede ser visto como problemático para la evaluación es lo que podría llamarse “desconexión en la implementación”, se refiere a que los planes usualmente no incluyen los medios para su propia implementación. Los planes confían en los instrumentos de implementación de aquellos sectores en los que intenta influir. El ejemplo del desarrollo de los parques eólicos marinos ilustra esta problemática. Es una de las fuentes de energía oceánica más nuevas y requiere la neutralización de grandes áreas marinas durante largos períodos de tiempo para la instalación de las turbinas eólicas (Carneiro, 2013).

Gee et al. (2019) distinguen a los sectores según su potencialidad de conflicto, es decir, denominan a las plataformas, ya sea de exploración y explotación de hidrocarburos, como a las de generación de energía renovable, "hard sector" (sector duro) y a los otros sectores que no involucran estas instalaciones, como la pesca, "soft sector" (sector blando). Los sectores "duros" requieren infraestructura fija en el mar; tienden a permanecer en el lugar durante mucho tiempo, su instalación es onerosa y son difíciles de mover. Los sectores "blandos" tienden a ser más móviles. La prevención de los conflictos es particularmente importante en el caso de los usos "duros", ya que es difícil modificar una situación luego de la instalación.

Gee et al. (2019) distinguen entre dos opciones para enfrentar los conflictos espaciales. La prevención del conflicto se centra en evitar la competencia espacial, usualmente asegurándose que las actividades incompatibles no ocurran en el mismo espacio o se afecten entre sí negativamente. La prevención se desarrolla antes de que se genere el conflicto y es anticipatoria por naturaleza. Por otro lado, la mitigación es aquella acción que busca suavizar los impactos de la competencia espacial. Es relevante en el caso de los conflictos inevitables- por ejemplo porque las decisiones de instalación ya se han tomado, o porque determinadas opciones espaciales pueden ya no estar disponibles, por ejemplo en espacios marinos que ya están muy ocupados. El objetivo principal de la mitigación consiste en suavizar los impactos de la competencia espacial, por ejemplo, por medio de medidas compensatorias negociadas entre los sectores afectados. Entre las acciones de mitigación pueden enumerarse los códigos voluntarios de conducta y la declaración de zonas de no acceso. 
Radovich, V.S. El mar también existe:reflexiones sobre la planificación espacial marina (PEM) como herramienta de gestión integral. Derecho y Ciencias Sociales. Mayo - Octubre 2020.N 23. Pgs 46-69 ISNN 1852-2971 Instituto de Cultura Jurídica y Maestría en Sociología Jurídica. FCJ y S. UNLP

Ehler \& Douvere (2009) señalan que desafortunadamente no se realiza suficiente trabajo sobre la geografía humana o social de los océanos y señalan que las dimensiones humanas del ambiente marino deberían ser incluidas e integradas en el proceso de toma de decisiones. Hay algunas capas de información socio-económica que deben combinarse con las capas de información biofísica, por ejemplo, en el análisis espacial para el establecimiento de un área marina protegida (AMP).

\section{Paso 6: Definir y analizar las condiciones futuras}

Los siguientes son los resultados que deberían obtenerse de esta etapa: un escenario de tendencia que ilustre cómo será el área de la PEM si continúan las condiciones actuales sin las nuevas intervenciones de gestión; usos alternativos espaciales del mar que ilustren cómo se verá el área de manejo cuando se redistribuyan las actividades humanas con base en las nuevas metas y objetivos; y un escenario de preferencia que provea la base para identificar y seleccionar las medidas de gestión en el plan de manejo espacial (Paso 7).

La PEM es una actividad orientada al futuro, su objetivo es ayudar a envisionar y crear un futuro deseable y permitir la toma de decisión proactiva a corto plazo para encaminarse hacia el objetivo deseado. En consecuencia, la planificación no debería limitarse a definir y analizar sólo las condiciones actuales y a mantener el status quo, sino que debería revelar posibles alternativas futuras de cómo podría verse el área en 10, 15 o 20 años (Ehler \& Douvere, 2009).

La República Federal de Alemania y los Países Bajos, por ejemplo, pudieron prever la cantidad de espacio que se requería para que todas las propuestas de la industria de la energía renovable en el mar pudieran ser operativas.

En el caso belga, todas las metas y objetivos fueron agrupados en tres categorías:

- Ecología y biodiversidad: esta categoría incluye las metas y los objetivos que contribuyen a la conservación y al mantenimiento del funcionamiento y la biodiversidad ecológica del área (por ej., objetivos relativos a la creación de AMPs); 
Radovich, V.S. El mar también existe:reflexiones sobre la planificación espacial marina (PEM) como herramienta de gestión integral. Derecho y Ciencias Sociales. Mayo - Octubre 2020.N 23. Pgs 46-69 ISNN 1852-2971 Instituto de Cultura Jurídica y Maestría en Sociología Jurídica. FCJ y S. UNLP

- Economía: esta categoría incluye las metas y los objetivos que contribuyen a las ganancias económicas que se obtienen del uso de los recursos marinos del área de gestión (por ej. objetivos relativos a maximizar el transporte marítimo en el área); y

- Sociedad y cultura: esta categoría incluye las metas y los objetivos que contribuyen al bienestar de la población en el área (por ej., objetivos relativos a la creación de oportunidades turísticas o sobre la preservación del patrimonio cultural).

Los objetivos de la PEM se resumen en dos campos: (1) la mejora del estatus marino ambiental, a través de la coordinación mejorada del desarrollo sectorial y medidas de gestión ambiental - por ejemplo cambiando de lugar las actividades que dañan el ambiente a áreas menos sensibles, o rediseñando medidas de impacto de mitigación para adaptarse mejor a la sensibilidad del área; y (2) la promoción de actividades marítimas, en particular su performance económica - por ej. La estrategia de "Crecimiento Azul" en Europaprincipalmente haciendo explícito para los inversores cómo se debe utilizar el espacio oceánico, mejorando la previsibilidad de inversiones y reduciendo los tiempos de licenciamiento (Carneiro, 2013).

\section{Paso 7: Preparar y aprobar el plan de manejo espacial}

Ehler \& Douvere (2009) describen los resultados que deberían obtenerse de este paso: la identificación y la evaluación de las medidas de manejo alternativo para el plan de manejo espacial; y un plan de gestión comprehensivo, que incluya si fuera necesario, un plan de zonificación.

Una medida de gestión espacial (y temporal) es una medida para producir los bienes y servicios deseados en un área de gestión marina que especifica cómo, dónde, y cuándo deben desarrollarse las actividades humanas, por ejemplo: la especificación de las áreas cercanas a la pesca u a otras actividades humanas; la designación de áreas precautorias o zonas de seguridad; la designación de AMPs; la zonificación de áreas para usos específicos; por ejemplo, parques eólicos, operaciones militares, minería de arena, eliminación de residuos, transporte marino, acuacultura; la zonificación de áreas por objetivo; por ejemplo, áreas de desarrollo, áreas de conservación, áreas de usos múltiples.

En general, el plan de gestión espacial debería incluir: la descripción de los límites del área de la PEM, así como una base específica anual y un período temporal del plan; las metas y 
Radovich, V.S. El mar también existe:reflexiones sobre la planificación espacial marina (PEM) como herramienta de gestión integral. Derecho y Ciencias Sociales. Mayo - Octubre 2020.N 23. Pgs 46-69 ISNN 1852-2971 Instituto de Cultura Jurídica y Maestría en Sociología Jurídica. FCJ y S. UNLP

los objetivos de manejo espacial; la descripción de las metas que se desean obtener en el futuro - un gráfico de la visión del desarrollo físico y de la conservación del área de manejo; las medidas de gestión requeridas para lograr el escenario futuro planteado; el cronograma sobre las acciones formales necesarias para implementar el plan (quién desarrolla qué actividades, dónde); y los requisitos de financiamiento del plan y el plan financiero que detalle las fuentes de financiamiento.

Actualmente, la mayoría de los países requieren una Evaluación Ambiental Estratégica (EAE) o Declaración de Impacto Ambiental (DIA) de los planes de gestión comprehensivos y de los programas públicos de inversión. La Directiva Europea (2001/42/EC) sobre la Evaluación de los Efectos de Ciertos Planes y Programas sobre el Ambiente ${ }^{8}$, por ejemplo, requiere una evaluación ambiental para ciertos planes y programas que puedan tener efectos significativos sobre el ambiente en varios niveles (nacional, regional y local).

\section{Paso 8: Implementar y ejecutar el plan espacial de manejo}

La implementación es el proceso de conversión de los planes de PEM en programas operativos, reales de funcionamiento (Ehler \& Douvere, 2009). Como parte del proceso de implementación, las instituciones públicas designadas o las nuevas entidades creadas (consejos coordinadores interministeriales) abordarán las nuevas acciones de gestión establecidas en el plan de gestión aprobado. La implementación es un paso de importancia crítica del proceso de la PEM. En general, los países no optan por la creación de una «súper» agencia de gestión marina (como sí ha decidido el Reino Unido, por ejemplo), se suele crear algún tipo de consejo interdisciplinario o interministerial, o una agencia «líder» designada para coordinar y supervisar el proceso de la PEM. El proceso será operativo cuando este régimen institucional empiece a funcionar de manera continuada. Las instituciones de gestión unisectoriales existentes llevarán a cabo la mayoría de las acciones para la implementación. Estas instituciones pueden usar el plan integral y el plan de zonificación como guías para los permisos, además de llevar a cabo otras actividades bajo su responsabilidad. La implementación también se puede coordinar entre distintos niveles de gobierno, esto sucederá en los países que cuentan con la forma de gobierno federal.

\footnotetext{
8 Directiva Europea (2001/42/EC) sobre la Evaluación de los Efectos de Ciertos Planes y Programas sobre el Ambiente del 27 de junio de 2001, Diario Oficial de las Comunidades Europeas L 197/30. Recuperado de https://www.boe.es/doue/2001/197/L00030-00037.pdf
} 
Radovich, V.S. El mar también existe:reflexiones sobre la planificación espacial marina (PEM) como herramienta de gestión integral. Derecho y Ciencias Sociales. Mayo - Octubre 2020.N 23. Pgs 46-69 ISNN 1852-2971 Instituto de Cultura Jurídica y Maestría en Sociología Jurídica. FCJ y S. UNLP

Además, hay que garantizar el cumplimiento del plan. Hay cumplimiento cuando se implementan los requisitos y se logran los cambios de comportamiento deseados de manera que, para dar algunos ejemplos, los límites de capturas no se exceden, las actividades humanas están emplazadas en lugares apropiados de las áreas designadas, o ciertas actividades humanas no se llevan a cabo en lugares protegidos. El cumplimiento es la implementación de los requisitos de la PEM. El cumplimiento y la ejecución son elementos esenciales del buen gobierno. Sin embargo, suelen ser los eslabones más débiles del proceso de PEM (Ehler \& Douvere, 2009).

El cumplimiento requerirá que todas las entidades de gestión unisectoriales responsables no sólo adopten estos planes mientras llevan a cabo sus propias tareas, sino que generen sus propios planes y programas en conformidad con el plan de gestión espacial.

La ejecución se refiere a la serie de acciones que los gobiernos llevan a cabo para lograr el cumplimiento de las regulaciones que involucran a las actividades humanas con el fin de corregir o detener las situaciones que hacen peligrar al ambiente o la comunidad.

Estas acciones por parte del Gobierno incluyen inspecciones, negociaciones con las partes interesadas y si proceden, acciones legales para garantizar el cumplimiento. Las ONG`s también pueden involucrarse en estas acciones.

Además, algunas industrias (por ejemplo, el sector bancario o de las aseguradoras) pueden estar indirectamente involucradas en la ejecución al exigir el cumplimiento de los requisitos de la PEM antes de aprobar un préstamo o una póliza de seguros para construir una planta en el mar.

Una tarea importante en relación con la ejecución consiste en asegurar que las estrategias, los planes y los reglamentos no sean demasiado restrictivos y además, se deben integrar transversalmente en todos los sectores, y se deben comunicar de una manera clara y concisa al público y al sector privado. Los actores suelen apoyar una ejecución efectiva si los reglamentos se aplican sobre la base de políticas y procedimientos transparentes.

\section{Paso 9: Monitorear y evaluar el cumplimiento del plan}

Los siguientes son los resultados que deben obtenerse de este paso: un sistema de monitoreo diseñado para medir indicadores de cumplimiento de las medidas de gestión espacial 
Radovich, V.S. El mar también existe:reflexiones sobre la planificación espacial marina (PEM) como herramienta de gestión integral. Derecho y Ciencias Sociales. Mayo - Octubre 2020.N 23. Pgs 46-69 ISNN 1852-2971 Instituto de Cultura Jurídica y Maestría en Sociología Jurídica. FCJ y S. UNLP

marina; información sobre la performance de las medidas de gestión especial marina que se utilizarán para la evaluación; y que se brinden informes periódicos a los tomadores de decisiones, grupos de interés, y al público sobre la performance del plan de manejo marino espacial (Ehler \& Douvere, 2009).

La evaluación ex post de la PEM ha recibido cierta atención (Day, 2008; Douvere \& Ehler, 2011; Carneiro, 2013), más que nada en el desarrollo de los métodos. Los académicos que se dedican a la planificación y los que la llevan a cabo suelen observar que el proceso de planificación es más importante que el plan en sí. Suele suceder que en el momento en que se aprueba un plan, se lo considera desactualizado principalmente porque el contexto en que se ha creado el plan ha cambiado - lo que justifica la planificación ininterrumpida (Carneiro, 2013).

\section{PASO 10: Adaptar el proceso de manejo espacial}

Los siguientes son los resultados que deberían obtenerse de este paso: propuestas para adaptar las metas, los objetivos, los resultados y las estrategias para la próxima ronda de planificación; la identificación de las necesidades de investigación aplicada.

Los resultados del monitoreo y de la evaluación se deben utilizar para adaptar la planificación y la gestión espacial marina para que sus acciones surtan los efectos deseados. La mayoría, si no todos los planes de gestión, tienen que revisarse y actualizarse regularmente (Ehler \& Douvere, 2009).

Un enfoque adaptativo implica explorar maneras alternativas para cumplir con los objetivos de la PEM, anticipar sus productos basándose en el estado actual del conocimiento, implementar una o más alternativas, monitorear estas alternativas para observar los efectos de las medidas de gestión y, por último, usar los resultados para ajustar las acciones de gestión.

Por ejemplo, en Australia, el Parque Marino del Gran Arrecife de Coral utilizó información del monitoreo y evaluación en su Programa de Áreas Representativas (1999-2004) para rezonificar e incrementar sus zonas de protección estricta desde el 5 por ciento al 33 por ciento de su área total. 
Radovich, V.S. El mar también existe:reflexiones sobre la planificación espacial marina (PEM) como herramienta de gestión integral. Derecho y Ciencias Sociales. Mayo - Octubre 2020.N 23. Pgs 46-69 ISNN 1852-2971 Instituto de Cultura Jurídica y Maestría en Sociología Jurídica. FCJ y S. UNLP

\section{Estudio de casos}

Se ha decidido comentar sobre el caso de China debido a que es uno de los primeros que ha regulado la PEM, también se decidió hacer una referencia al continente sudamericano, ya que es donde el desarrollo de la PEM es más incipiente.

La documentación de las experiencias de la PEM suele ser útil como una forma de aprendizaje. Es importante documentar cómo se resolvieron los problemas específicos en un contexto en particular, para que puedan ser comparados y evaluados (Carneiro, 2013)

En Europa, se ha conformado una Comisión sobre las mejores prácticas internacionales para la PEM transfronteriza que emite recomendaciones basadas en la revisión de cuatro procesos de PEM que se desarrollaron fuera de Europa: la Isla de Rodas, el Triángulo de Coral, el Océano del Sur y Xiamen. Algunas de las buenas prácticas identificadas incluyen: comprender profundamente el sistema de gobernanza actual; invertir tiempo y recursos durante el proceso de PEM para construir confianza y un sentido de logro del objetivo en común; adoptar un enfoque basado en torno a la PEM; adoptar una perspectiva a largo plazo; gestionar las expectativas para el involucramiento de los grupos de interés; y diseñar un sistema de monitoreo y evaluación que analice el cumplimiento y el progreso hacia los objetivos (EC DG MARE, UNESCO IOC, 2018).

En el ámbito asiático, existe un proyecto transfronterizo de investigación sobre PEM entre la República Popular China y Corea del Sur en el Mar Amarillo. La República Popular China ha estado trabajando en la PEM desde 1979 - a través del manejo costero integrado (MCI) y está preparando actualmente el cuarto plan de generación. En cambio, el trabajo sobre la PEM en Corea del Sur es más reciente, ya que el primer Plan nacional fue adoptado en el año 2018, el mismo año en que se sancionó la ley coreana sobre planificación espacial marina y se fundó el Centro para la Gestión y la Evaluación de la Planificación Espacial Marina (CMSPAM, por sus siglas en coreano). La experiencia reveló un número de desafíos relativos a: el gran tamaño del Mar Amarillo, los diferentes marcos de políticas, las disputas regionales sobre límites marinos, la limitada disponibilidad de datos y los conflictos de intereses entre los grupos de intereses nacionales (Zhiwei, 2018).

En China, la Administración Estatal del Océano (SOA, por sus siglas en inglés) administra la denominada Zonificación funcional marina (MFZ, por sus siglas en inglés). SOA redacta las leyes y las regulaciones concernientes a los usos de las zonas del océano, a la protección ambiental y a la investigación científica. 
Radovich, V.S. El mar también existe:reflexiones sobre la planificación espacial marina (PEM) como herramienta de gestión integral. Derecho y Ciencias Sociales. Mayo - Octubre 2020.N 23. Pgs 46-69 ISNN 1852-2971 Instituto de Cultura Jurídica y Maestría en Sociología Jurídica. FCJ y S. UNLP

La MFZ, es una herramienta de zonificación de múltiples usos de la planificación espacial marina, que se desarrolla en China desde hace treinta años. La MFZ fue propuesta por primera vez en China en 1988, y ya en 1989 se establecieron unidades experimentales en el Mar Bohai.

En 1997, como respuesta a la política nacional de China concerniente a la protección ambiental de los recursos naturales, SOA propuso oficialmente la elaboración de la Ley sobre Gestión Oceánica, que fue adoptada en 2001 por el Congreso y entró en vigor en 2002. Para realizar algún uso en el Océano, previamente se debe pedir autorización al Estado Nacional, que es el propietario del Océano y se debe pagar una tarifa.

La implementación de la MFZ en China ha revelado ciertas debilidades que son similares a las que se observan en otros países, la necesidad de mejorar la: (1) coordinación entre la planificación del océano y la tierra; (2) resolución de conflictos entre los usos humanos y el ambiente marino; (3) la evaluación y el monitoreo del proceso; y (4) participación de los grupos de interesados en el proceso de zonificación ${ }^{9}$.

Con respecto al Extenso Ecosistema Marino del Caribe y al extenso ecosistema (LME, por sus siglas en inglés) de la Plataforma Norte de Brasil, referidas colectivamente como CLME+, hasta ahora, los esfuerzos para la implementación de la PEM siguen siendo nacionales y no transfronterizos. Se ha detectado en la región que las disputas entre los sectores, por ejemplo entre el sector de la pesca y el ambiente, hacen peligrar la implementación de la PEM.

Una de las buenas prácticas que se sugiere es que los grupos de interés se involucren en un diálogo constructivo, así los sectores podrían comprender más adecuadamente los puntos de vista de cada uno y construir confianza. Se identificó un número de elementos que ayudan a emprender satisfactoriamente los procesos de PEM: la voluntad política, el conocimiento y la práctica, el liderazgo, el uso efectivo de los fondos, la existencia de un marco legal, la participación de los grupos de interesados (Díaz de León, 2018).

Otra de las buenas prácticas que se identificaron consisten en que la comunicación y la interconsulta son importantes para organizar reuniones cara a cara en una etapa temprana e invertir en reuniones frecuentes con los grupos de interesados. Es probable que algún grupo

\footnotetext{
${ }^{9}$ La aplicación de la PEM en China. Recuperado de http://msp.ioc-unesco.org/world-applications/asia/china/
} 
Radovich, V.S. El mar también existe:reflexiones sobre la planificación espacial marina (PEM) como herramienta de gestión integral. Derecho y Ciencias Sociales. Mayo - Octubre 2020.N 23. Pgs 46-69 ISNN 1852-2971 Instituto de Cultura Jurídica y Maestría en Sociología Jurídica. FCJ y S. UNLP

de interesados desee unirse al proceso en una etapa posterior a su propia iniciativa y nunca es demasiado tarde para involucrarlos, se recomienda ser flexible en este sentido.

Para intentar prevenir conflictos entre los grupos de interesados, el proceso de planificación debe comenzar con el desarrollo de una matriz de grupos de interesados, para mapear todos los posibles conflictos y entender el poder y la responsabilidad de cada parte.

Con respecto a la región sudamericana, Echevarría, Gómez, Gómez \& Tejera (2015) al referirse a la experiencia uruguaya, sostienen que la exploración de hidrocarburos en el mar podría aprovecharse para realizar la PEM en el país y comentan que el Ministerio de Relaciones Exteriores del país ha querido liderar el proceso. Sostienen que debido a que Uruguay comparte parte del espacio marítimo con la República Argentina es necesaria la coordinación para generar un Plan Marítimo Transfronterizo común para el Río de la Plata y su frente marítimo.

En la República Argentina, en mayo de 2019 se otorgaron permisos para la exploración y explotación de hidrocarburos en el mar a través de la Resolución 276/19 de la Secretaría de Gobierno de Energía sin realizar previamente las audiencias o consultas públicas ni las evaluaciones de impacto ambiental exigidas por la LGA y no se ha comenzado el proceso de planificación espacial marina en el país.

\section{Conclusiones}

El objetivo del presente artículo consistió en generar conocimiento sobre la planificación espacial marina (PEM), un instrumento enmarcado en la gestión integral del mar que propone abordar las actividades que se desarrollan en el espacio marino coordinadamente, no de manera sectorial como se ha hecho históricamente.

Se describió el proceso de pasos que propone la UNESCO para implementar la PEM y ciertas críticas que se han hecho a este método, que alegan que en realidad el proceso de la PEM no suele ser participativo y que suele ser sectorial, no integral como indica su nombre.

La PEM se implementa porque existen o se prevé que existan usos incompatibles o usos que afecten adversamente importantes áreas naturales. Los problemas suelen estar relacionados con la compatibilización entre el desarrollo económico y la conservación del ambiente. 
Radovich, V.S. El mar también existe:reflexiones sobre la planificación espacial marina (PEM) como herramienta de gestión integral. Derecho y Ciencias Sociales. Mayo - Octubre 2020.N 23. Pgs 46-69 ISNN 1852-2971 Instituto de Cultura Jurídica y Maestría en Sociología Jurídica. FCJ y S. UNLP

En la mayoría de las iniciativas a nivel mundial sobre la PEM, se suele designar una nueva autoridad para desarrollarla, mientras que la implementación se lleva adelante mediante autoridades e instituciones ya existentes.

Se describieron ciertas fortalezas, debilidades y desafíos futuros que se vislumbraron en los estudios de casos. Por ejemplo, en América del Sur, Argentina y Uruguay deberían desarrollar un plan marítimo transfronterizo para el Río de la Plata.

Algunas de las buenas prácticas identificadas en los estudios de casos analizados incluyen: comprender profundamente el sistema de gobernanza actual; invertir tiempo y recursos durante el proceso de PEM para construir confianza y un sentido de logro del objetivo en común; adoptar un enfoque basado en torno a la PEM; adoptar una perspectiva a largo plazo; gestionar las expectativas para el involucramiento de los grupos de interesados; y diseñar un sistema de monitoreo y evaluación que analice el cumplimiento y el progreso hacia los objetivos.

Otra de las buenas prácticas que se identificaron es que los grupos de interesados deberían involucrarse en un diálogo constructivo, así los sectores podrían comprender más adecuadamente los puntos de vista de cada uno y construir confianza. Es crucial convencer a los grupos de interés sectoriales acerca de que pueden encontrar sinergias entre ellos y que no constituyen necesariamente una amenaza para el otro. Se identificó una serie de elementos que ayudan a emprender satisfactoriamente los procesos de PEM: la voluntad política, el conocimiento y la práctica, el liderazgo, el uso efectivo de los fondos y la existencia de un marco legal.

También se concluye que el proceso de planificación es más importante que el plan en sí, ya que suele suceder que en el momento en que se aprueba el plan, se lo considere desactualizado, por lo que la planificación debe ser flexible e ininterrumpida.

\section{Bibliografía}

Carneiro, G. (abril, 2013). Evaluation of marine spatial planning: selected challenges and experiences. Documento presentado en el EEEN Forum. Malmo, Suecia.

De Carvalho, J. (mayo, 2018). Marine Spatial Planning in the Bengela Current LME. Documento presentado en el EC DG MARE and UNESCO IOC International Forum for Marine Spatial Planning. Bruselas, Bélgica. 
Radovich, V.S. El mar también existe:reflexiones sobre la planificación espacial marina (PEM) como herramienta de gestión integral. Derecho y Ciencias Sociales. Mayo - Octubre 2020.N 23. Pgs 46-69 ISNN 1852-2971 Instituto de Cultura Jurídica y Maestría en Sociología Jurídica. FCJ y S. UNLP

Díaz de León, V. (mayo, 2018). Caribbean Large Marine Ecosystem. Documento presentado en el EC DG MARE and UNESCO IOC International Forum for Marine Spatial Planning. Bruselas, Bélgica.

EC DG MARE, UNESCO IOC (2018). Informe del Primer Foro Internacional de Planificación Espacial Marina. Bruselas, Bélgica. Recuperado de https://www.mspplatform.eu/sites/default/files/20180731_report.pdf

Echevarría, L., Gómez, A., Gómez, M. \& Tejera, F. (2015). La planificación espacial marina como herramienta de gestión. Montevideo, Uruguay: Colección Interdisciplinarias. Universidad de la República.

Ehler, C. \& Douvere, F. (2009). Planificación espacial marina: una guía paso a paso hacia la gestión ecosistémica. París, Francia: Comisión Oceanográfica Intergubernamental y el Programa del Hombre y la Biosfera. COI manuales y guías no 53 UNESCO. 2009 (inglés). 2013 (español).

Gee, K., et al. (2019). Addressing conflicting spatial demands in MSP. Considerations for MSP planners. Luxemburgo, Luxemburgo: Oficina de Publicaciones de la Unión Europea.

Iglesias Campos, A. (mayo 2018). International Guidelines on Marine Spatial Planning. Documento presentado en el EC DG MARE and UNESCO IOC International Forum for Marine Spatial Planning. Bruselas, Bélgica.

IPBES (2019). Global assessment report on biodiversity and ecosystem services of the Intergovernmental Science- Policy Platform on Biodiversity and Ecosystem Services. Chapter 6. E. S. Brondizio, J. Settele, S. Díaz, and H. T. Ngo (editors). Bonn, Germany: IPBES Secretariat.

Jones, P., Lieberknecht, L. \& Qiu, W. (2016). Marine spatial planning in reality: Introduction to case studies and discussion of findings. Marine Policy 71, 256-264. doi:10.1016/j.marpol.2016.04.026.

Mabilia, V. (mayo, 2018). Cross border Maritime Spatial Planning. Documento presentado en el EC DG MARE and UNESCO IOC International Forum for Marine Spatial Planning. Bruselas, Bélgica.

OECD (2016). The Ocean Economy in 2030. Paris: OECD Publishing. DOI: http://dx.doi.org/10.1787/9789264251724-en

Radovich, V. (2018). Implicancias ambientales de la exploración y la explotación hidrocarburífera en el mar con especial referencia al régimen de la República Argentina y del MERCOSUR (tesis doctoral). Universidad de Buenos Aires. Facultad de Derecho. Buenos Aires, Argentina: Repositorio Digital Institucional de la Universidad de Buenos Aires http://repositoriouba.sisbi.uba.ar/gsdl/cgibin/library.cgi?e=q-10000-00---off-0adrposgra--00-2----0-10-0---0---0direct-10--ZZ--4------0-11--10-es-Zz-1---20-about-violeta+radovich--00-3-1-00-00--4--0--0-0-01-00OutfZz-800\&amp;a=d\&amp;c=adrposgra\&amp;srp=0\&amp;srn=0\&amp;cl=search\&amp;d=HW A_2760 
Radovich, V.S. El mar también existe:reflexiones sobre la planificación espacial marina (PEM) como herramienta de gestión integral. Derecho y Ciencias Sociales. Mayo - Octubre 2020.N 23. Pgs 46-69 ISNN 1852-2971 Instituto de Cultura Jurídica y Maestría en Sociología Jurídica. FCJ y S. UNLP

Waldmann, C. et al. (2019). Cross border consultation on maritime spatial plans. Luxemburgo, Luxemburgo: Oficina de Publicaciones de la Unión Europea.

Zhiwei, Z. (mayo, 2018). Collaborative Research Interests in Maritime Spatial Planning in Yellow Sea and Progress between China and Korea. Documento presentado en el EC DG MARE and UNESCO IOC International Forum for Marine Spatial Planning. Bruselas, Bélgica.

\section{Legislación:}

Argentina. Ley 25.675, Ley General del Ambiente. Boletín Oficial, 28/11/2002.

Argentina. Resolución 276/19. Secretaría de Gobierno de Energía. Boletín Oficial, 17/05/2019.

Australia. The Great Barrier Reef Marine Park Act, 1975, Federal Register of Legislation C2017C00279. Recuperado de https://www.legislation.gov.au/Details/C2017C00279

China. Ley sobre Gestión Oceánica, 2002.

Convención sobre la Diversidad Biológica (“CDB”), adoptada 5 junio de 1992, 1760 UNTS 79 (entró en vigor 29 de diciembre de 1993).

EE.UU. Oceans Act of 2008, Chapter 114, Commonwealth of Massachusetts, United States of America. Recuperado de https://malegislature.gov/Laws/SessionLaws/Acts/2008/Chapter114

Organización de las Naciones Unidas, 2015. Agenda de Desarrollo Sostenible 2030. Recuperado de https://www.onu.org.ar/agenda-post-2015/

Reino Unido. The Marine and Coastal Acess Act, del 12 de noviembre de 2009, c. 23. Recuperado de http:// www.defra.gov.uk/marine/legislation/index.htm

Unión Europea. Directiva Europea (2001/42/EC) sobre la Evaluación de los Efectos de Ciertos Planes y Programas sobre el Ambiente del 27 de junio de 2001, Diario Oficial de las Comunidades Europeas L 197/30. Recuperado de https://www.boe.es/doue/2001/197/L00030-00037.pdf

Unión Europea. Directiva 2014/89/EU del Parlamento Europeo y del Consejo del 23 de julio de 2014, por la que se establece un marco para la ordenación del espacio marítimo, Diario Oficial de la Unión Europea L 257/135. Recuperado de https://eurlex.europa.eu/legal-content/ES/TXT/PDF/?uri=CELEX:32014L0089\&from=EN

Jurisprudencia:

"Villivar, Silvia Noemí, c/ Provincia de Chubut y otros", (2007 CSJN, Fallos: 330:1791). 